\title{
NOUVELLE
}

\section{Une vaccination efficace en dose unique contre la fièvre de Lassa}

Sylvain Baize

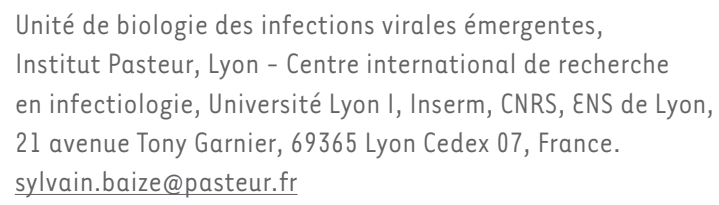

> La fièvre de Lassa est causée par le virus Lassa (LASV), un Arénavirus de I'ancien monde. Elle se manifeste par une fièvre hémorragique, mortelle chez $15 \%$ à $30 \%$ des patients hospitalisés. La fièvre de Lassa est endémique en Afrique de l'Ouest, et est responsable de plusieurs dizaines de milliers de cas par an et plusieurs milliers de décès [1]. De plus, c'est la fièvre hémorragique la plus fréquemment importée dans les pays du Nord, avec 1 à 2 cas annuels en moyenne, et une transmission secondaire en Allemagne il y a quelques années. Aucun traitement efficace ou vaccin autorisé n'est disponible à ce jour pour lutter contre cette maladie récemment listée par l'Organisation mondiale de la santé (OMS) comme maladie émergente nécessitant le développement en urgence de contre-mesures. LASV se transmet à l'homme par l'intermédiaire de son réservoir naturel, majoritairement le rongeur péri-domestique Mastomys natalensis ${ }^{l}$. Ensuite, une transmission interhumaine par le biais de contacts cutanés ou muqueux peut survenir. La proximité entre le réservoir et l'homme est

${ }^{1}$ Mastomys est un genre de rongeurs africains de la sousfamille des murinés. 
à l'origine de fréquentes introductions de LASV en zone d'endémie, avec un pic en saison sèche [2]. Les réponses lymphocytaires $T$ semblent cruciales pour le contrôle de l'infection par LASV, tant au cours de l'infection naturelle que dans le cadre d'une vaccination expérimentale, mais il est possible que les anticorps jouent également un rôle $[3,4]$. Les caractéristiques épidémiologiques de la fièvre de Lassa font d'une approche vaccinale le moyen idéal de lutter efficacement contre cette infection grave, qui constitue un problème majeur de santé publique dans les pays d'Afrique de l'Ouest. Le principal obstacle pour produire un vaccin efficace est la grande diversité génétique qui existe parmi les souches de LASV. À cela s'ajoute la difficulté de vacciner l'ensemble de la population dans des pays disposant de ressources limitées, et dont une grande partie vit dans des zones reculées et difficiles d'accès, ce qui plaide en faveur de l'obtention d'un vaccin efficace en une seule injection. Quelques candidats vaccins ont montré une bonne efficacité, parfois après une seule injection. Certains sont fondés sur des vecteurs vivants atténués, comme ML29, un virus recombinant dont le génome est composé d'un segment du génome de LASV et d'un autre issu du virus Mopeia (MOPV), un Arénavirus proche de LASV mais non pathogène pour les primates non-humains, ou comme le virus de la stomatite vésiculaire (VSV) exprimant le précurseur de la glycoprotéine (GPC) de LASV [4, 5]. Bien que ces vaccins soient efficaces après administration d'une dose unique, leur innocuité reste cependant à démontrer. Une autre approche utilise un vaccin $A D N$, mais nécessite trois injections vaccinales pour que celui-ci soit efficace [6]. Nous avons récemment développé un vaccin contre les Arénavirus fondé sur un MOPV hyperatténué exprimant la GPC d'un Arénavirus pathogène et porteur de mutations dans le gène codant la nucléoprotéine (NP) abrogeant la capacité du virus à inhiber la réponse interféron (IFN) de type I (MOPEVAC) [7]. Nous avons également utilisé le vaccin contre la rougeole dans lequel nous avons inséré d'une part les gènes codant la GPC et la NP du LASV contenant les mutations abrogeant ses propriétés immunosuppressives (MeV-NP), et, d'autre part, les gènes codant la GPC et la protéine de matrice Z du LASV (MeV-Z) $[8,9]$. Afin de sélectionner le meilleur vaccin parmi ces trois candidats (MOPEVAC, MeV-NP, et MeV-Z), nous avons comparé leur immunogénicité et leur efficacité chez le singe cynomolgus (macaque à longue queue), le modèle animal le plus pertinent pour étudier la fièvre de Lassa [3]. Tous ont été très bien tolérés et ont protégé les animaux contre l'infection par le LASV et la fièvre de Lassa après une seule injection, mais avec une efficacité variable [9]. MeV-Z s'est avéré peu efficace. En effet, les animaux ayant reçu ce vaccin et soumis à un challenge infectieux avec le LASV ont présenté des scores cliniques élevés, une fièvre persistante, et un contrôle médiocre de la réplication virale. L'un d'entre eux présentait encore un score clinique proche du point limite un mois après l'infection, et une charge virale infectieuse dans les organes lymphoïdes secondaires. MOPEVAC s'est en revanche avéré très efficace, 3 animaux sur 4 n'ayant présenté qu'une fièvre modérée pendant quelques jours et une virémie très faible et transitoire. Le quatrième est demeuré asymptomatique, sans aucune trace de réplication virale, indiquant une immunité stérilisante. Des résultats très satisfaisants ont également été obtenus avec MeV-NP. Les scores cliniques ont été quasiment nuls, marqués seulement par une fièvre de courte durée, sans aucune altération des fonctions hépatique et rénale. Des traces d'ARN viraux, sans virus infectieux, ont été détectées dans le plasma, mais uniquement six jours après l'infection. Les candidats MOPEVAC et surtout MeV-NP sont donc des vaccins très efficaces, en dose unique, contre LASV.

Les paramètres de la réponse immunitaire impliqués dans le contrôle de l'infection par le LASV n'étant pas complètement identifiés, nous avons dis- séqué les réponses immunitaires innée, humorale et cellulaire, après l'immunisation et le challenge infectieux. Tous les animaux vaccinés par MOPEVAC ont synthétisé des immunoglobulines G (IgG) spécifiques du LASV avant le challenge infectieux, et la plupart ont développé des titres modérés d'anticorps neutralisants. La moitié des animaux vaccinés par MeV-NP présentaient des IgG dirigées contre le LASV avant l'infection, et un seul avait des anticorps neutralisants. En revanche, ces réponses étaient absentes dans le groupe des animaux vaccinés par MeV-Z. Des réponses lymphocytaires $T$ $\mathrm{CD}^{+}$et $\mathrm{CD} 8^{+}$spécifiques des GPC et de NP étaient présentes après l'immunisation chez les animaux vaccinés avec MOPEVAC et MeV-NP, mais pas ou peu avec MeV-Z. Les profils transcriptomiques des cellules mononucléées sanguines périphériques (peripheral blood mononuclear cells, PBMC) ont également été analysés, et ont révélé une transcription intense des gènes de réponse aux IFN de type I dès deux jours après l'immunisation dans les groupes de singes vaccinés par MOPEVAC ou par MeV-NP, tandis que cette réponse était de faible intensité et retardée chez les animaux vaccinés par MeV-Z. Alors que cette surexpression génique n'était observée que deux jours après l'immunisation avec MOPEVAC, elle persistait une semaine avec MeV-NP, suggérant une réponse innée plus durable pour ce dernier. Le challenge infectieux avec le LASV a provoqué une augmentation très forte des réponses immunitaires humorale et cellulaire chez les animaux vaccinés. Des taux élevés d'IgG dirigées contre le LASV et d'anticorps neutralisants ont été mesurés respectivement 9 et 15 jours après le challenge infectieux. Le pourcentage de lymphocytes $\mathrm{TCD}^{+}$et $\mathrm{CD}^{+}$produisant des cytokines de type Thl (IFN- $\gamma$ et TNF- $\alpha$ ) a fortement augmenté dès 9 jours après l'infection chez les animaux vaccinés par MOPEVAC ou MeV-NP, mais pas chez ceux vaccinés par $\mathrm{MeV}-Z$, ni chez les animaux témoins non vaccinés. Afin de préciser les paramètres de la réponse immunitaire impliqués 
dans la protection induite par la vaccination, nous avons corrélé chacun de ces paramètres avec le score clinique et la charge virale plasmatique mesurés tout au long de l'infection (Figure 1). Le titre d'anticorps neutralisants ne semble pas être associé à un meilleur contrôle de l'infection chez les animaux. De même, les réponses des lymphocytes $T$ spécifiques de la NP ne sont pas corrélées à des signes cliniques et à une charge virale plus modérés. En revanche, le taux d'IgG non neutralisantes spécifiques du LASV et les réponses des lymphocytes T $\mathrm{CD}^{+}$et plus particulièrement des $\mathrm{CD} 8^{+}$ spécifiques de la GPC sont inversement corrélés à la sévérité de l'infection. Ces résultats suggèrent que l'induction de lymphocytes $\mathrm{T} \mathrm{CD} 4^{+}$et $\mathrm{CD} 8^{+}$spécifiques de la GPC ainsi que les anticorps non neutralisants seraient majoritairement impliqués dans le contrôle de l'infection par le LASV. Un tel rôle des IgG dirigées contre le LASV a été récemment suggéré pour un candidat vaccin fondé sur le virus de la rage [10]. Il est néanmoins probable que la présence de la NP dans le vaccin soit importante pour la protection vaccinale, si l'on en croit le gradient de protection observé avec le vaccin n'exprimant pas la NP (MeV-Z), peu efficace, celui exprimant une NP hétérologue (MOPEVAC), et celui exprimant la NP du LASV (MeV-NP), le plus efficace. De même, d'autres études ont montré que si la GPC était l'antigène indispensable pour qu'un vaccin contre la fièvre de Lassa soit protecteur, l'ajout de la NP en potentialisait l'efficacité. Dans notre étude, deux vaccins vivants atténués exprimant les NP et GPC du LASV induisent une protection robuste après une seule injection. Le plus efficace, MeV-NP, est actuellement en cours d'évaluation chez l'homme dans le cadre d'un essai clinique de phase l, et un essai de phase II devrait suivre très prochainement. Nous avons également démontré récemment que MeV-NP était capable de protéger des singes cynomolgus contre des souches très divergentes de LASV, et que les animaux vaccinés avec une seule dose étaient toujours parfaitement protégés un an après l'injection du vaccin.
Une vaccination de masse, avec un vaccin efficace en dose unique, des dizaines de millions de personnes à risque pour l'infection par le LASV permettrait de limiter considérablement l'incidence de la fièvre de Lassa dans les pays d'Afrique où elle est endémique, et d'atténuer le poids important que cette maladie représente en termes de santé publique. Le vaccin MeV-NP a été construit à partir du vaccin contre la rougeole, dont l'innocuité et l'efficacité ne sont plus à démontrer, et il est donc un candidat idéal pour atteindre cet objectif, d'autant plus qu'il a été clairement démontré que la présence d'une immunité préexistante contre la rougeole n'empêchait pas le vaccin d'induire une réponse immunitaire robuste contre les antigènes exogènes exprimés par ce vaccin. $\diamond$

\section{A single shot vaccine against Lassa fever}

\section{LIENS D'INTÉRÊT}

L'auteur déclare n'avoir aucun lien d'intérêt concernant les données publiées dans cet article.

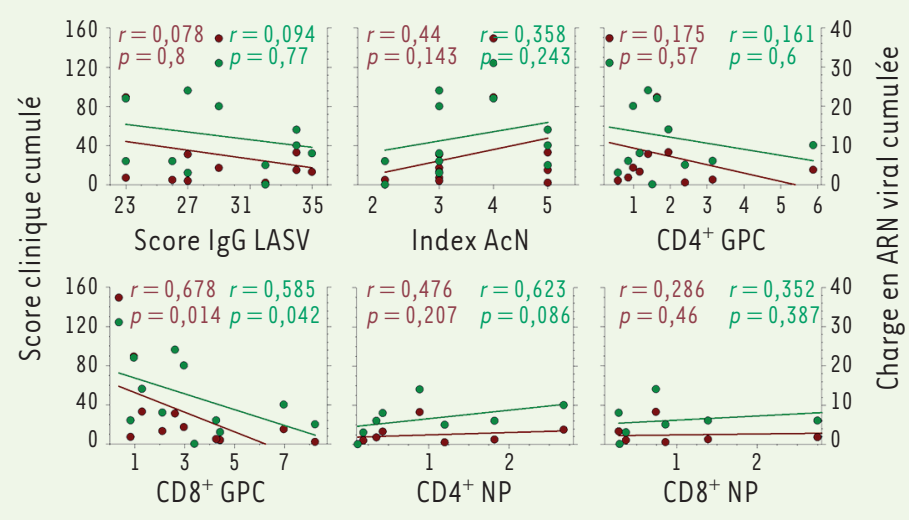

Figure 1. Association entre les paramètres de la réponse immune et l'efficacité de la protection contre la fièvre de Lassa. Les réponses immunes humorales et cellulaires spécifiques du virus Lassa (LASV) sont représentées en fonction du score clinique (axe vertical gauche et points rouges) et de la charge en ARN viral (axe vertical droit et points verts) cumulés. Les scores cliniques mesurés quotidiennement ont été additionnés jusqu'à 28 jours après le challenge infectieux. La charge en ARN viral cumulée a été calculée en additionnant les valeurs obtenues $3,6,9,12$, 15,22 et 28 jours après le challenge ( 1 si CV

comprise entre le seuil et $2 \times 10^{4}$ copies $/ \mathrm{mL} ; 2$ si comprise entre $2 \times 10^{4}$ et $3 \times 10^{4}, 3$ entre $3 \times 10^{4}$ et $5 \times 10^{4}, 4$ entre $5 \times 10^{4}$ et $10 \times 10^{4}, 5$ entre $10 \times 10^{4}$ et $25 \times 10^{4}, 6$ entre $25 \times 10^{4}$ et $5 \times 10^{5}, 8$ entre $5 \times 10^{5}$ et $10^{6}$, et 10 si supérieure à $\left.10^{6}\right)$. Pour le score d'IgG dirigées contre le LASV, les densités optiques (D0) obtenues pour tous les échantillons de chaque animal (7, 10, 14, 21 et 30 jours après l'immunisation et $3,6,9,12,15,22$ et 28 jours après le challenge) ont été additionnées selon le code : 1 si la D0 est comprise entre la valeur seuil et 0,$05 ; 2$ si comprise entre 0,05 et 0,$1 ; 3$ entre 0,1 et 0,$2 ; 4$ entre 0,2 et 0,$35 ; 5$ entre 0,35 et 0,$5 ; 6$ entre 0,5 et 0,$7 ; 8$ entre 0,7 et 1,0 ; et 10 si supérieure à 1.0 . L'index d'anticorps neutralisants $(\mathrm{AcN})$ a été calculé de manière similaire, avec 1 correspondant à un titre de 1:100 et 2 pour 1:500. Les pourcentages de lymphocytes T produisant de l'interféron $\gamma$ (IFN- $\gamma$ ) et/ou tumor necrosis factor $\alpha$ (TNF- $\alpha$ ) ont été additionnés pour tous les échantillons de chaque animal obtenus après l'immunisation et le challenge infectieux avec le LASV aux mêmes temps que pour la réponse humorale. Les droites de régression linéaire sont montrées pour chaque paramètre et score clinique (droites rouges) ou charge virale (droites vertes), ainsi que le coefficient de corrélation et la valeur des $p$ respectifs. Les différents scores obtenus pour les paramètres de la réponse immunitaire sont représentés sur l'axe des abscisses. 


\section{RÉFÉRENCES}

1. McCormick JB, Webb PA, Krebs JW, et al. A prospective study of the epidemiology and ecology of Lassa fever. $J$ Infect Dis 1987 ; 155 : 437-44.

2. Kafetzopoulou LE, Pullan ST, Lemey P, et al. Metagenomic sequencing at the epicenter of the Nigeria 2018 Lassa fever outbreak. Science 2019 $363: 74-7$.

3. Baize $S$, Marianneau $P$, Loth $P$, et al. Early and strong immune responses are associated with control of viral replication and recovery in Lassa virus-infected cynomolgus monkeys. J Virol $2009 ; 83: 5890-903$.
4. Geisbert TW, Jones S, Fritz EA, et al. Development of a new vaccine for the prevention of Lassa fever. PLoS Med $2005 ; 2: 537-45$.

5. Carrion JR, Patterson JL, Johnson C, et al. A ML29 reassortant virus protects guinea pigs against a distantly related Nigerian strain of Lassa virus and can provide sterilizing immunity. Vaccine 2007 ; 25 : 4093-102.

6. Cashman KA, Wilkinson $\varepsilon R$, Shaia $\mathrm{Cl}$, et al. A DNA vaccine delivered by dermal electroporation fully protects cynomolgus macaques against Lassa fever. Hum Vacc Immunother 2017 ; 13 : 2902-11.

7. Carnec X, Mateo M, Page A, et al. A vaccine platform against Arenaviruses based on a recombinant hyperattenuated Mopeia virus expressing heterologous glycoproteins.J Virol 2018 ; 92 : e02230-17.

8. Frantz PN, Teeravechyan S, Tangy F. Measles-derived vaccines to prevent emerging viral diseases. Microb Infect 2018; $20: 493-500$.

9. Mateo M, Reynard S, Carnec X, et al. Vaccines inducing immunity to Lassa virus glycoprotein and nucleoprotein protect macaques after a single shot. Sci Transl Med 2019; 11:eaaw3163.

10. Abreu-Mota T, Hagen KR, Cooper K, et al. Nonneutralizing antibodies elicited by recombinant Lassa-Rabies vaccine are critical for protection against Lassa fever. Nat Commun 2018 ; 9 : 4223
NOUVELLE

\section{Un rôle pour les astrocytes dans les déficiences intellectuelles?}

${ }^{1}$ Interactions neurogliales dans

la physiologie et pathologies cérébrales, Centre interdisciplinaire de recherche

en biologie, Collège de France, CNRS

UMR 7241, Inserm U1050, Labex Memolife, Université PSL, 75005 Paris, France.

${ }^{2}$ Institut de psychiatrie et de neurosciences de Paris, Inserm U1266, université de Paris, 75014 Paris, France.

*Contribution égale

pierre.billuart@inserm.fr

nathalie.rouach@college-de-france.fr

> Les troubles du neurodéveloppement, qui incluent les déficiences intellectuelles, se caractérisent par des anomalies dans la formation et le fonctionnement des circuits synaptiques. Bien que la plupart des recherches sur la synapse dans le domaine de la santé et des maladies soient restreintes à l'étude des neurones, un nombre croissant de travaux souligne l'importance des astrocytes dans la mise en place et la fonction des synapses. Ainsi, quelques études portant sur les déficiences intellectuelles ont récemment mis en évidence les contributions potentielles des astrocytes dans la pathogenèse de ces affections. Dans cet article, nous analysons comment les altérations des fonctions astrogliales dans la maladie peuvent affecter la formation ou le fonctionnement des synapses et l'excitabilité neuronale, et ainsi contribuer à la déficience intellectuelle.

La neuropathie : une vision restrictive des déficiences intellectuelles

La déficience intellectuelle (également appelée trouble d'apprentissage, retard mental, ou déficit cognitif) est définie par un quotient intellectuel ( $\mathrm{QI}$ ) inférieur à 70 , associé à un déficit des capacités d'adaptation conceptuelle, sociale et pratique se manifestant avant l'âge de 18 ans. Les causes de la déficience intellectuelle sont hétérogènes et incluent des facteurs génétiques et environnementaux (infections, intoxications, etc.), qui interfèrent avec le développement et le fonctionnement du système nerveux central durant la période périnatale. Les causes génétiques sont responsables de $40 \%$ à $50 \%$ des déficiences intellectuelles modérées à sévères (quotient intellectuel $<50$ ), les plus connues étant la trisomie 21, le syndrome de Rett (mutations du gène MECP2 codant la methyl-CpG-binding protein 2), et le syndrome du chromosome $X$ fragile (mutations du gène FMRI codant la FMRP translational regulator 1 ). La plupart des gènes impliqués dans les déficiences intellectuelles codent des protéines enrichies dans les compartiments synaptiques, et qui sont impliquées dans le développement des circuits neuronaux [1]. L'expression de ces gènes, initialement considérée comme restreinte aux neurones, a récemment été décrite dans les astrocytes, suggérant que ces cellules gliales pourraient également contribuer à la déficience intellectuelle [2].
Notre analyse récente de l'expression de quelque 600 gènes impliqués dans la déficience intellectuelle ne montre en effet pas d'enrichissement global dans un type cellulaire donné (Figure IA). Une analyse approfondie portant sur 1370 gènes impliqués dans la déficience intellectuelle révèle même que 361 d'entre eux (groupe 2 de la Figure IB) sont plus exprimés dans les astrocytes que dans les neurones [3, 4]. II convient de noter que les orthologues murins de FMRI ou $M E C P 2$, ainsi que ceux de plusieurs gènes impliqués dans la trisomie 21 (SODI, DYRKIa, S100 $\beta$ et OLIG2), sont également exprimés dans les astrocytes (Figure IA).

\section{Perturbations du développement} et du phénotype des astrocytes dans la déficience intellectuelle Alors que la plupart des déficiences intellectuelles et des troubles du spectre autistique sont présentés comme des anomalies du développement neuronal, le développement des astrocytes y est également affecté. Des études portant sur la trisomie 21 ont notamment montré une augmentation du nombre d'astrocytes et de leur complexité morphologique, 